\title{
Characteristics and Treatment of Cutaneous Melanoma of the Foot
}

\author{
Kyung Wook Nam ${ }^{1}$, Yong Chan Bae ${ }^{1,2}$, Soo Bong Nam ${ }^{1}$, Joo Hyung Kim ${ }^{1}$, Hoon Soo Kim², \\ Young Jin $\mathrm{Choi}^{4}$ \\ ${ }^{1}$ Department of Plastic and Reconstructive Surgery, Pusan National University School of Medicine, Busan; ${ }^{2}$ Biomedical Research Institute, \\ Pusan National University Hospital, Busan; Departments of ${ }^{3}$ Dermatology and ${ }^{4}$ Internal Medicine, Pusan National University School of \\ Medicine, Busan, Korea
}

Background In East Asia, the foot is the most common site of cutaneous melanoma. The purpose of this study was to investigate the differences between cutaneous melanoma of the foot and melanomas of other sites.

Methods We studied 52 patients who underwent surgical treatment for cutaneous melanoma of the foot from September 2000 to January 2015. Through a retrospective review of their medical records, we collected data relating to their sex, age, histopathological subtype, Clark level, tumor thickness, lymph node involvement, stage, and survival rate, and we compared these parameters to those of 61 patients treated for melanoma of other sites. Moreover, we analyzed the surgical procedures, complications, and mortality rates associated with cutaneous melanoma on various parts of the foot.

Results Melanoma of the foot differed from other melanomas only in the histopathological subtype. Other clinical features, including survival rate, did not differ significantly between the two groups heel was the most common site of melanoma of the foot (22 cases, 42.3\%). The method of reconstruction varied depending on the region involved.

Conclusions A comparison of melanoma of the foot to other melanomas showed that melanoma of the foot was associated with a significantly different distribution of histological subtypes. Consistent with previous findings, we found that the acral lentiginous subtype was the most common among melanomas of the foot. Furthermore, in contrast with previous studies, we did not find a worse prognosis or lower survival rate for melanoma of the foot in comparison with other melanomas.

Keywords Foot / Melanoma / Skin neoplasms
Correspondence: Yong Chan Bae Department of Plastic and Reconstructive Surgery, Pusan National University School of Medicine, 179 Gudeok-ro, Seo-gu, Busan 49241, Korea

Tel: +82-51-240-7273

Fax: +82-51-243-9405

E-mail: baeyc2@hanmail.net

This work was supported by a two-year research grant from Pusan National University.

No potential conflict of interest relevant to this article was reported.

\section{INTRODUCTION}

Malignant melanoma is the most common cause of death from skin cancer [1-3]. The incidence rate and clinical features of its subtypes differ significantly according to biological (particularly race and sex) and environmental factors [4-6].

The primary site of malignant melanoma varies significantly among racial groups [4]. In general, the head and neck region and the trunk region are the most common sites of melanoma in Caucasian males, and the lower extremity region is the most 
common site for Caucasian females. In these populations, melanoma occurs on the foot and toes very rarely. However, in East Asia, melanoma occurs most commonly on the foot.

Many studies have shown that ultraviolet light exposure, multiple benign or atypical nevi, immunosuppression, and sun sensitivity may cause malignant melanoma [7-9]. In addition, previous trauma or nevi, chemical exposure, and smoking have been reported to be risk factors for melanoma of the hand and foot $[10,11]$.

It has been suggested that the etiology of melanoma of the foot may be different from that of other melanomas. Recently, this issue has attracted significant attention, including studies involving gene mutation pattern analysis and a wide range of clinical studies.

As the first stage of this research program, we attempted to evaluate the distinguishing features of cutaneous melanoma of the foot, in comparison to melanomas that occur on other parts of the body. Each melanoma was classified according to its site on the foot, and the treatment strategies, complications, and survival rates were investigated. Our analysis may serve as a reference point for determining treatment strategies in the future.

\section{METHODS}

\section{Subjects}

The subjects of this study were patients who underwent surgical treatment for cutaneous melanoma from September 2000 to January 2015. A group of 52 patients with melanoma of the foot (group A) were compared to 61 patients with melanoma of other sites (group B). The other sites included the head and neck (15 cases), the upper extremity (20 cases), other locations on the lower extremity (13 cases), and the trunk (13 cases).

\section{Research methods}

Through a retrospective review of the patients' medical records, we recorded the sex and age of each patient, the histopathological subtype, Clark level, tumor thickness, lymph node involvement, and stage of each tumor, calculated the survival rate, and compared 52 patients with melanoma of the foot to 61 patients with melanoma of other sites.

Age was treated as a continuous variable, and the clinical histopathological subtypes were divided into lentigo maligna melanoma, superficial spreading melanoma, acral lentiginous melanoma, and nodular melanoma.

The tumor thickness was measured in millimeters and treated as another continuous variable. Lymph node involvement was determined by the presence of invasion. The stages of melanoma were defined using the 2013 National Comprehensive Can- cer Network guidelines, based on the pathological histology of the lesion and the clinical record of the patient at the time of diagnosis.

Group A and group B were compared using the chi-squared test for the parameters involving sex, age, clinical histopathological type, Clark level, tumor thickness, lymph node involvement, tumor stage, and survival rate, while the continuous variables (age and tumor thickness) were examined using Wilcoxon's signed rank test in order to assess whether significant differences were present between the two groups with regard to each variable.

The Kaplan-Meier method was used to estimate the survival rate of groups $\mathrm{A}$ and $\mathrm{B}$, and the log-rank test was used to compare the survival rate of groups $A$ and $B$.

Group A, which included patients with cutaneous melanoma of the foot, was further divided into subgroups depending on whether the melanoma occurred on the toe, heel, midsole, medial side, or lateral side, and each sub-group was evaluated according to the patient's age, clinical histopathological type, Clark level, tumor thickness, lymph node involvement, and tumor stage. In addition, we investigated the complications associated with each operative technique.

\section{Treatment protocol for melanoma}

The basic treatment plan for malignant melanoma was wide local excision and reconstruction with lymph node evaluation.

We adopted wide local excision margins depending on the Breslow thickness. The surgical margin was $0.5 \mathrm{~cm}$ for in situ melanoma, $1 \mathrm{~cm}$ for lesions $\leq 1 \mathrm{~mm}$ in depth, $2 \mathrm{~cm}$ for lesions $1.01-2.0 \mathrm{~mm}$ in depth, and $3 \mathrm{~cm}$ for lesions $>2.0 \mathrm{~mm}$ in depth [12].

In order to determine whether regional lymph node metastasis occurred, which is an important prognostic factor, a sentinel lymph node biopsy was carried out if the tumor thickness was at least $1 \mathrm{~mm}$. If the patient had risk factors for lymph node involvement, such as ulceration or a high mitotic rate, tumors less than $1 \mathrm{~mm}$ in thickness likewise underwent sentinel lymph node biopsy. Lymph node dissection was performed if the biopsy result was positive.

In some cases in the initial period of the study, insufficient clinical cooperation with sentinel lymph node biopsy resulted in the performance of direct lymph node dissection without biopsy.

The reconstruction methods after the wide local excision were free flaps, local flaps, skin grafts, and sural flaps, according to the specific site of the melanoma and the defect size.

Adjuvant therapy was initiated depending on the patient's condition and tumor stage, based on consultation with the hematology/oncology internal medicine clinic. 


\section{RESULTS}

\section{Characteristics of patients with malignant melanoma of the foot}

\section{Sex and age}

The female-to-male ratio was 1:1.48 in group $\mathrm{A}$ and 1:2.05 in group B. The overall female-to-male ratio was 1:1.76. The mean age of the patients at the time of diagnosis was $63 \pm 11.0$ years in group A and $59 \pm 16.2$ years in group B. Overall, the mean age of the two groups was $61 \pm 14.2$ years (Table 1). No significant differences in sex $(\mathrm{P}=0.403)$ or age $(\mathrm{P}=0.057)$ were observed between the two groups.

\section{Histopathological subtype}

Malignant melanoma was classified clinically and pathologically. In group $\mathrm{A}$, most cases were acral lentiginous melanoma (42 cases, $80.8 \%$ ), and in group B, nodular melanoma was the most common subtype (23 cases, 37.7\%). Regarding the distribution of histopathological subtypes, a statistically significant difference was observed between the two groups $(\mathrm{P}<0.001)$ (Table 2).

\section{Level of invasion}

Clark level IV was the most common level of invasion in both groups, and no statistically significant difference was observed between the two groups in this regard (Table 3).

\section{Tumor thickness}

The Breslow thickness classification (in situ, $\leq 1 \mathrm{~mm}, 1.01-2$ $\mathrm{mm}, 2.01-4 \mathrm{~mm},>4 \mathrm{~mm}$ ) is presented in Table 4 . The average

\section{Table 1. Distribution of malignant melanoma patients by} sex and mean age

\begin{tabular}{|lccc|}
\hline Sex/age & Foot & Others & Total \\
\hline No. of patients (\%) & $52(46.0)$ & $61(54.0)$ & $113(100)$ \\
Male & 21 & 20 & 41 \\
Female & 31 & 41 & 72 \\
Female-to-male ratio & 1.5 & 2.1 & 1.8 \\
Mean age & $63.9 \pm 10.9$ & $59.0 \pm 16.2$ & $61.3 \pm 14.2$ \\
\hline
\end{tabular}

Table 2. Distribution of histopathological subtypes among malignant melanoma patients

\begin{tabular}{|lccc|}
\hline Subtype & Foot & Others & Total \\
\hline Acral lentiginous & $45(86.6)$ & $17(26.2)$ & $58(51.3)$ \\
Lentigo maligna & - & $8(6.6)$ & $4(3.5)$ \\
Nodular & $7(13.5)$ & $26(37.7)$ & $30(26.6)$ \\
Superficial spreading & - & $10(14.8)$ & $9(8.0)$ \\
Total & 52 & 61 & 113 \\
\hline Values are presented as number (\%). & & \\
\hline
\end{tabular}

Breslow thickness in group A was $4.47 \pm 4.5 \mathrm{~mm}$, and $4.63 \pm 7.2$ $\mathrm{mm}$ in group $\mathrm{B}$. The difference between the two groups was not statistically significant $(\mathrm{P}=0.568)$.

\section{Lymph node metastasis}

Lymph node metastasis was observed in 19 cases (36.5\%) in group A and 29 cases (31.1\%) in group B. The difference between the two groups was not statistically significant $(\mathrm{P}=0.676)$.

\section{Staging}

In both groups A and B, the plurality of tumors were stage II. No significant difference was observed between the two groups in this regard $(\mathrm{P}=0.658)$ (Table 5).

\section{Survival rate}

Ten patients (17.3\%) passed away in group A, and 10 patients (18.0\%) passed away in group B. The two groups did not show

\section{Table 3. Distribution of melanoma cases by the Clark level}

\begin{tabular}{|lccc|}
\hline Clark's level & Foot & Others & Total \\
\hline I & $9(17.3)$ & $7(11.5)$ & $16(14.2)$ \\
II & $5(9.6)$ & $5(8.2)$ & $10(8.9)$ \\
III & $5(9.6)$ & $14(23.0)$ & $19(16.8)$ \\
IV & $20(38.5)$ & $21(34.4)$ & $41(36.3)$ \\
V & $13(25.0)$ & $14(23.0)$ & $27(23.9)$ \\
Total & 52 & 61 & 113 \\
\hline Values are presented as number (\%). & & \\
\hline
\end{tabular}

Table 4. Distribution of melanoma cases by the thickness of the melanoma

\begin{tabular}{|lccc|}
\hline Thickness $(\mathrm{mm})$ & Foot & Others & Total \\
\hline In situ & $8(15.4)$ & $6(9.8)$ & $14(12.4)$ \\
$\leq 1$ & $6(11.5)$ & $9(14.8)$ & $15(13.3)$ \\
$1.01-2$ & $4(7.7)$ & $10(16.4)$ & $14(12.4)$ \\
$2.01-4$ & $14(26.9)$ & $17(27.9)$ & $31(27.4)$ \\
$>4$ & $20(38.5)$ & $19(31.2)$ & $39(34.5)$ \\
Total & 52 & 61 & 113 \\
\hline Values are presented as number (\%). & & \\
\hline
\end{tabular}

\section{Table 5. Distribution of melanoma cases by stage}

\begin{tabular}{|lccc|}
\hline Stage & Foot & Others & Total \\
\hline 0 & $8(15.4)$ & $6(9.8)$ & $14(12.4)$ \\
1 & $9(17.3)$ & $11(16.4)$ & $20(16.8)$ \\
2 & $17(26.9)$ & $22(27.9)$ & $39(27.4)$ \\
3 & $15(15.4)$ & $21(29.5)$ & $36(23.0)$ \\
4 & $3(25.0)$ & $1(16.4)$ & $4(20.4)$ \\
Total & 52 & 61 & 113 \\
\hline Values are presented as number (\%). & & \\
\hline
\end{tabular}


a statistically significant difference in the survival rate $(\mathrm{P}=0.938)$ (Fig. 1).

\section{Results of treatment for melanoma of the foot}

The most common site of melanoma of the foot was the heel (22 cases), followed by the sole ( 14 cases) and the toe ( 11 cases). Four cases were on the lateral side of the foot, and one case was on the medial side (Table 6, Fig. 2).

The free flap method was used in 12 cases, the local flap method was used in 15 cases, split-thickness skin grafts were used in

\section{Fig. 1. Survival rates of foot and other areas}

The two groups did not show a significantly different survival rate $(P=0.938)$. (A) Skin lesions of the foot, (B) skin lesions of other areas.

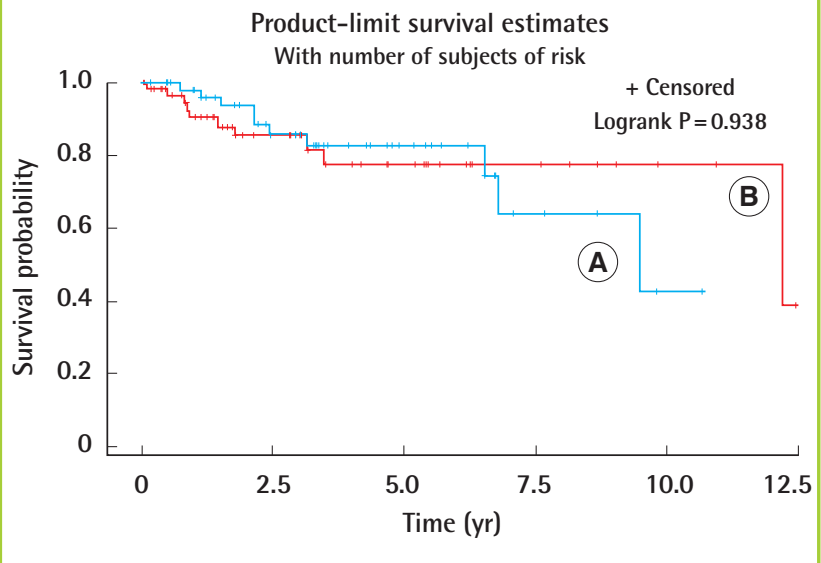

11 cases, and sural flaps were used in 14 cases.

Eight cases involved complications, including wound dehiscence in two cases, chronic ulceration in one case, and gait discomfort in five cases (Table 7).

Twenty-two of the 51 patients received adjuvant therapy. Four patients received interferon-alpha (IFN- $\alpha$ ) only, and five patients received dacarbazine (DTIC) chemotherapy only. Three patients were treated with both IFN- $\alpha$ and DTIC. Ten patients passed away from malignant melanoma.

\section{Fig. 2. Distribution of sites of foot melanoma}

The most common site of melanoma of the foot was the heel (22 cases), followed by the sole (11 cases) and the toe (11 cases).
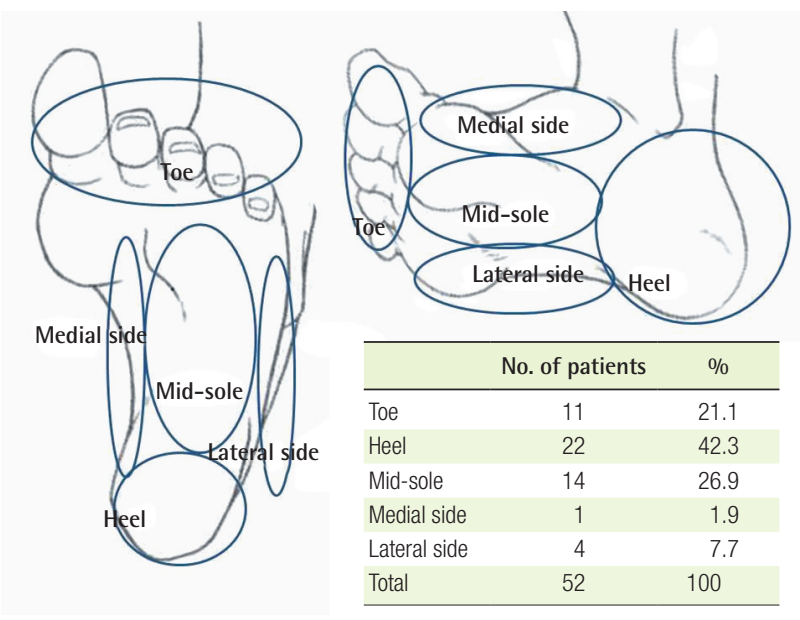

Table 6. Characteristics of the specific sites of melanoma of the foot

\begin{tabular}{|c|c|c|c|c|c|}
\hline Characteristic & Toe & Heel & Mid-sole & Medial side & Lateral side \\
\hline Sex & 11 & 22 & 14 & 1 & 4 \\
\hline Male & 3 & 8 & 8 & 1 & 2 \\
\hline Female & 8 & 14 & 6 & & 2 \\
\hline Age (yr) & $68.0 \pm 11.0$ & $65.0 \pm 11.0$ & $59.3 \pm 10.2$ & 72.0 & $61.3 \pm 11.0$ \\
\hline \multicolumn{6}{|l|}{ Subtype } \\
\hline Acral lentiginous & 10 & 18 & 12 & 1 & 4 \\
\hline Nodular & 2 & 4 & 1 & & \\
\hline \multicolumn{6}{|l|}{ Clark level } \\
\hline 1 & 2 & 3 & 4 & & \\
\hline 2 & 1 & 3 & 1 & & \\
\hline 3 & 3 & 1 & 1 & & \\
\hline 4 & 2 & 7 & 6 & 1 & 4 \\
\hline 5 & 3 & 8 & 2 & & \\
\hline Depth (mm) & $4.0 \pm 3.1$ & $6.1 \pm 6.0$ & $2.2 \pm 2.4$ & 3.5 & $4.9 \pm 1.7$ \\
\hline LN involvement (\%) & $5(45.5)$ & $4(18.2)$ & $6(42.9)$ & & $4(100)$ \\
\hline \multicolumn{6}{|l|}{ Stage } \\
\hline 0 & 2 & 3 & 2 & & \\
\hline 1 & 1 & 3 & 5 & & \\
\hline 2 & 5 & 7 & 3 & & 1 \\
\hline 3 & 2 & 7 & 3 & & 3 \\
\hline 4 & 1 & 1 & 1 & 1 & \\
\hline No. of expired (\%) & $1(9.1)$ & $5(22.7)$ & $4(28.6)$ & & \\
\hline
\end{tabular}


Table 7. Reconstruction methods and complications based on the specific sites of melanoma of the foot

\begin{tabular}{|c|c|c|c|c|c|c|c|c|}
\hline & \multirow{2}{*}{ Toe } & \multirow{2}{*}{ Heel } & \multirow{2}{*}{ Mid-sole } & \multirow{2}{*}{$\begin{array}{l}\text { Medial } \\
\text { side }\end{array}$} & \multirow{2}{*}{$\begin{array}{l}\text { Lateral } \\
\text { side }\end{array}$} & \multicolumn{3}{|c|}{ Complications } \\
\hline & & & & & & Gait discomfort & Wound dehiscence & Chronic ulcer \\
\hline No. of patients & 11 & 22 & 14 & 1 & 4 & - & - & - \\
\hline Free flap & - & 5 & 7 & - & - & - & - & - \\
\hline Local flap & 11 & 1 & 2 & 0 & 1 & 2 & 1 & - \\
\hline STSG & - & 2 & 5 & 1 & 3 & - & - & 1 \\
\hline Sural flap & - & 14 & - & - & - & 3 & 1 & - \\
\hline
\end{tabular}

\section{DISCUSSION}

The primary site of malignant melanoma differs significantly according to race. For non-Caucasians, such as East Asians, Blacks, and indigenous inhabitants of the Americas, the most common lesion sites are the hand-and-foot and nail regions [4-6]. Those regions have also been found to be the most common lesion sites in Koreans, comprising $58.6 \%$ and $61.5 \%$ of cases of malignant melanoma in two different studies $[1,2]$. In particular, the foot has been established as the most common site of melanoma in East Asian populations; similarly, Park et al. [3] found that the foot was the most common tumor site (48.5\%) among the subjects analyzed in their study.

Moreover, patients with malignant melanoma of the head and neck are often older than those with malignant melanoma of the trunk. This finding has led to the hypothesis that distinct etiologic pathways exist for melanoma depending on the anatomic site. Thus, we attempted to evaluate the clinical features of melanoma of the foot, based on the hypothesis that it may have different causes than other melanomas.

The incidence rate of malignant melanoma in this study was not significantly different among men and women in either group A or group B. Moreover, foot melanoma had the highest incidence rate among both males and females. However, in a study analyzing Korean subjects, Park et al. [13] found that the most common site for melanoma in men was the lower extremity, whereas it was the upper extremity in women, which was not the case for the subjects included in our study.

In group A, women had more cases of melanoma (59.6\%); Gray et al. [14] likewise reported more cases of malignant melanoma of the foot in women (58\%). However, the overall percentage of female patients was higher in this study, and no other Korean studies have presented findings regarding the sex ratio of patients with melanoma of the foot. Therefore, additional studies would be required to elucidate this question more conclusively.

The mean age of the patients in group A was 64 years, and that of the patients in group B was 59 years. The most common his- topathological subtype in Group A was acral lentiginous melanoma, whereas nodular melanoma was the most common subtype in group B. Jang et al. [1] reported that nodular melanoma and acral lentiginous melanoma occur mainly in patients in their 60s and late 50s. In their study, acral lentiginous melanoma and nodular melanoma accounted for $94 \%$ of cases of melanoma of the foot, compared to $64 \%$ of cases of melanoma of other sites, which was similar to our results. Gray et al. [14] reported that the mean age of patients with malignant melanoma was 61 years and that acral lentiginous melanoma was the most common subtype, comprising $42 \%$ of the cases analyzed in their study. However, additional studies may be required to determine whether the onset age of melanoma of the foot differs from the onset age of melanoma of other sites. After distant metastasis to the lung, brain, and bone, the condition of patients deteriorates, leading to death caused by malignant melanoma.

No significant difference in mortality was observed between group A and group B. Many studies conducted in Western countries $[10,15,16]$ have asserted that melanoma of the foot occupies a large portion of tissue and that aggressive resection is necessary due to frequent local and regional recurrence. A poor prognosis compared to melanoma of other parts of the body has also been reported. Bristow et al. [17] reported that patients failed to recognize sole or subungual melanomas, leading to a delayed diagnosis and poor prognosis. However, no differences regarding the outcome were found in this study. Additional research on gender differences in mortality due to malignant melanoma in various regions will likely be required.

The most common tumor site in the foot was the heel. Durbec et al. [10] reported that the most common site was on the sole, and Ishihara et al. [18] reported the same result. Jang et al. [1] reported that the middle of the sole was the most common site, followed by the heel. Considering the difficulty of identifying a clear boundary between the heel and sole, it would be logical to assume that most cases of melanoma of the foot occur on the sole.

The reconstruction method varied depending on the part of the foot involved. Local flaps are usually used in the distal third of the foot, and when the lesion is limited to the toe, local flap 
reconstruction using a skin envelope is performed after toe or ray amputation. We have also performed surgical repair using local flaps in cases involving the toe.

For defects in the midsole of the plantar midfoot, when the defect is small and is on the medial side of the arch in the nonweight bearing region, a skin graft is sufficient. However, if other regions are involved, flap surgery is required because most defects are large due to the necessity of wide local excision. Free flaps are considered to the proper technique for covering large defects, because they lowers donor site morbidity.

However, if the patient is in a relatively poor condition or recurrence is anticipated, a local flap and skin graft could be performed. The heel belongs to the plantar hind foot and plays an important role as a weight-bearing region and in ankle motion. The restoration of both form and function is important. The reconstruction methods used in the heel include medial plantar artery flaps, heel pad flaps, sural artery flaps, free flaps, and similar techniques. However, in the case of medial plantar artery flaps, the skin graft should be performed at the instep that is the donor site of the skin graft. If the defect is too large, such a technique cannot be applied. Flaps cannot be used in the heel pad to treat large defects.

We used a sural artery flap or a free flap in most cases, with one case of a local flap in a patient with a small defect and two cases of skin graft in patients with no possible ambulation. The medial side and the lateral side of the foot are not weight-bearing portions of the foot and do not influence ambulation, and those areas were therefore reconstructed using a local flap or skin graft [19].

If the lesion was limited to the toe, some cases were reconstructed with a local flap together with a skin envelope after toe or ray amputation. No cases required foot amputation or below-theknee amputation.

Currently, sentinel lymph node biopsy is considered the standard procedure for evaluating lymph node involvement, but immediate lymph node dissection was performed in some cases in the past. Forty-nine of the 52 patients in our study underwent lymph node evaluation; 24 patients underwent sentinel lymph node biopsy only, 10 patients underwent lymph node dissection immediately, and 15 patients underwent lymph node dissection after sentinel lymph node biopsy. Lymph node involvement was found in 19 patients. All four cases with melanoma on the lateral side of the foot showed lymph node involvement. However, the small number of cases is a limitation of this study, and further observation would be helpful.

Adjuvant therapy, primarily using IFN- $\alpha$, may be considered in patients with stage IIB-III melanoma who have a high risk of recurrence. If needed, adjuvant radiation therapy may also be initiated. We developed our therapeutic plans in consultation with the hematology and oncology department, and patients with stage IIB-IIIB melanoma were treated with IFN- $\alpha$. Cases of stage IIIC melanoma were considered to be high-risk, and DTIC chemotherapy was therefore immediately administered to those patients. When recurrence occurred despite adjuvant IFN- $\alpha$ therapy, additional DTIC chemotherapy was initiated. With regard to adjuvant therapy, no study has exclusively focused on melanoma of the foot, and only a few single-center studies have been reported. Additional multicenter studies should be conducted nationally.

The characteristics and the detailed site distribution of melanoma of the foot presented in this study may be useful in selecting treatment strategies and operative methods. Also, in contrast to previous findings, the prognosis of melanoma of the foot was found to be similar to that of melanoma of other sites, with no significant difference in survival rates observed between the two groups analyzed in our study.

\section{REFERENCES}

1. Jang KA, Kim JH, Choi JH, et al. A Clinico-histopathological study of malignant melanoma. Korean J Dermatol 2000; 38:1435-43.

2. Choi SJ, Bae YC, Moon JS, et al. An analysis of clinical and histopathological pattern of malignant melanoma. J Korean Soc Plast Reconstr Surg 2007;34:557-61.

3. Park KD, Lee SJ, Lee WJ, et al. clinicopathological features of cutaneous malignant melanoma. Korean J Dermatol DE 2007;45:149-58.

4. Reintgen DS, McCarty KM Jr, Cox E, et al. Malignant melanoma in black American and white American populations: a comparative review. Jama 1982;248:1856-9.

5. Kukita A, Ishihara K. Clinical features and distribution of malignant melanoma and pigmented nevi on the soles of the feet in Japan. J Invest Dermatol 1989;92:210s-213s.

6. Collins RJ. Melanoma in the Chinese of Hong Kong. Emphasis on volar and subungual sites. Cancer 1984;54:1482-8.

7. Juzeniene A, Baturaite Z, Moan J, et al. Sun exposure and melanomas on sun-shielded and sun-exposed body areas. In: Reichrath J, editor. Sunlight, vitamin D and skin cancer. New York: Springer; 2013. p.1-15.

8. Miller AJ, Mihm MC Jr. Melanoma. N Engl J Med 2006; 355:51-65.

9. Gilchrest BA, Eller MS, Geller AC, et al. The pathogenesis of melanoma induced by ultraviolet radiation. $\mathrm{N}$ Engl J Med 1999;340:1341-8.

10. Durbec F, Martin L, Derancourt C, et al. Melanoma of the 
hand and foot: epidemiological, prognostic and genetic features. A systematic review. Br J Dermatol 2012;166:727-39.

11. Green A, McCredie M, MacKie R, et al. A case-control study of melanomas of the soles and palms (Australia and Scotland). Cancer Causes Control 1999;10:21-5.

12. Coit DG, Andtbacka R, Anker CJ, et al. Melanoma, version 2.2013: featured updates to the NCCN guidelines. J Natl Compr Canc Netw 2013;11:395-407.

13. Park DH, Seo SJ, Park MC, et al. Clinical study of malignant melanoma for recent 14 years. J Korean Soc Plast Reconstr Surg 2009;36:299-305.

14. Gray RJ, Pockaj BA, Vega ML, et al. Diagnosis and treatment of malignant melanoma of the foot. Foot Ankle Int 2006;27: 696-705.

15. Sanlorenzo M, Osella-Abate S, Ribero S, et al. Melanoma of the lower extremities: foot site is an independent risk factor for clinical outcome. Int J Dermatol 2015;54:1023-9.

16. Talley LI, Soong S, Harrison RA, et al. Clinical outcomes of localized melanoma of the foot: a case-control study. J Clin Epidemiol 1998;51:853-7.

17. Bristow IR, de Berker DA, Acland KM, et al. Clinical guidelines for the recognition of melanoma of the foot and nail unit. J Foot Ankle Res 2010;3:25.

18. Ishihara K, Saida T, Otsuka F, et al. Statistical profiles of malignant melanoma and other skin cancers in Japan: 2007 update. Int J Clin Oncol 2008;13:33-41.

19. Mark WC, Lawrence BC, Christopher EA, et al. Foot reconstruction. In: Neligan P, Warren RJ, Van Beek A, editors. Plastic surgery. Philadelpia: Elsevier Saunders; 2011. p.207-17. 